Military Technical College Kobry El-Kobbah, Cairo, Egypt

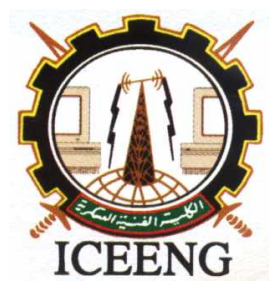

\section{$6^{\text {th }}$ International Conference on Electrical Engineering ICEENG 2008}

\title{
Antenna array design for low earth observation satellites
}

\author{
By \\ H. H. El-Banna* \\ A. A. Mitkees** \\ A. M. Allam ** \\ M. M. Mokhtar** \\ M. A. Zayan***
}

\section{Abstract:}

A rectangular planar array antenna design using thinning technique and Genetic Algorithm (GA) optimization technique are used to minimize the gain variation for Low Earth Orbit (LEO) satellites antenna. This design controls the feeding amplitude through thinning without requirements to control the phases which yields to simplify the feeding network.

Two antenna elements are proposed, $\cos ^{1.5} \theta$ radiation pattern element and the rectangular patch element.

A simple genetic algorithm optimizer is used to get the optimum excited elements on the array.

\section{Keywords:}

Antenna Arrays, LEO Satellite Antennas, Genetic Algorithm 


\section{Introduction:}

In the interests of achieving isoflux illumination, constant link margin and compensate the high slant range path-loss variation at low earth orbits from nadir to edge of coverage (EOC) [1] this paper presents a simple synthesis technique.

Thinning an array means turning off some elements in a uniformly spaced or periodic array to create a desired amplitude density across the aperture. An element connected to the feed network is "on", and an element connected to a matched or dummy load is "off". Thinning an array is one of the simplest excitation techniques to produce the desired antenna radiation pattern [2].

In order to achieve isoflux illumination and constant link margin, antenna gain must increase as a function of the angle away from nadir on the earth surface.

The required directivity may be achieved through changing the excitations of the elements by thinning or nonuniform excitations, and the beam was steered to the desired angle by using the appropriate phase shifts between the elements.

But, the aim of this paper is to present a more simple design to be easy in fabrication, which is to achieve the desired directivity only by amplitude excitations with no phase difference between the elements, so, no phase shifters are needed.

Two radiation antenna elements are proposed, $\cos ^{1.5} \theta$ (which can simplify other antenna elements) and the rectangular patch element.

A simulation results from $4 \times 4,8 \times 4$, and $8 \times 8$ rectangular planar arrays are discussed to achieve the required directivity from nadir till $58^{\circ}$, near the EOC [3].

Genetic algorithm $[4,5]$, has a high ability in global optimization. It is an increasingly popular optimization method being applied to many fields, including electromagnetic optimization problems. Using genetic algorithm to synthesize array pattern has no limitation on lattice and aperture shapes. It can synthesize planar array with arbitrary geometry and generating arbitrary patterns. Compared with other numerical methods $[6,7]$, this approach has unique features to treat complicated problems as arrays.

A genetic algorithm optimizer was built to get all of the optimum distribution of the ON and OFF elements, to achieve the optimum directivity through a specific ground track of a sun-synchronous orbit.

\section{Problem formulation:}

An x-band planar array configuration antenna as shown in Figure (1) is designed to be placed on a surface of a three axis stabilized cubic sun-synchronous satellite; the face which faced to the earth. The objective from this work is to adjust the antenna gain to compensate the path losses slant range variations from nadir till the EOC to achieve a constant link margin by a simple technique.

The far-field radiation pattern $\mathrm{F}(\theta, \phi)$ at an angle from the array broadside is given 
by [8]:

$\mathrm{F}(\theta, \phi)=\mathrm{EF}(\theta) \cdot \operatorname{AF}(\theta, \phi)$

where,

$\mathrm{EF}(\theta)= \begin{cases}\cos ^{1.5}(\theta) & \text { For the assumed element radiation pattern } \\ \cos \left(\frac{\pi}{2 \sqrt{\varepsilon_{\mathrm{r}}}} \cdot \sin (\theta)\right) & \text { For the half wave rectangular microstrip patch }\end{cases}$

is the normalized element radiation pattern in case of the assumed $\cos ^{1.5}(\theta)$ and the half wavelength rectangular microstrip antenna [9], $\varepsilon_{\mathrm{r}}$ is the dielectric constant taken equals 2.2 .

$$
\operatorname{AF}(\theta, \phi)=\sum_{n=1}^{N} \sum_{m=1}^{M} a_{m n} e^{j k\left[m d_{x} u+n_{y} v\right]}
$$

is the array radiation pattern, described by the following parameters;

$\mathrm{M}, \mathrm{N}=$ number of elements in the $\mathrm{x}$ and $\mathrm{y}$-direction, respectively,

$\mathrm{a}_{\mathrm{mn}}= \begin{cases}0 & \text { element OFF } \\ 1 & \text { element ON }\end{cases}$

$\mathrm{d}_{\mathrm{x}}=$ separation distance between two successive elements in the $\mathrm{x}$-direction,

$\mathrm{d}_{\mathrm{y}}=$ separation distance between two successive elements in the y-direction,

$\mathrm{u}=\sin \theta \cos \phi, \quad \mathrm{v}=\sin \theta \sin \phi$,

As the satellite go away w.r.t. the ground station the slant range is increased, so, the antenna directivity should be increased to keep fixed link margin, as follows:

$\mathrm{DD}_{\mathrm{r}}(\theta, \phi)=\mathrm{D}_{0}\left[\frac{\mathrm{R}_{\mathrm{s}}(\theta, \phi)}{\mathrm{h}}\right]^{2}$

where:

$\mathrm{DD}_{\mathrm{r}}$ : the desired directivity (ratio) at each slant range,

$\mathrm{D}_{0}$ : the required directivity (ratio) at nadir, is taken equal $3 \mathrm{~dB}$.

$h$ : the satellite altitude,

$R_{s}$ : the slant range from the earth station to the satellite, calculated as follows: 


$$
\mathrm{R}_{\mathrm{s}}=\sqrt{-2 \mathrm{R}_{\mathrm{e}}\left(\mathrm{R}_{\mathrm{e}}+\mathrm{h}\right) \cos (\gamma)+\left(\mathrm{R}_{\mathrm{e}}+\mathrm{h}\right)^{2}+\mathrm{R}_{\mathrm{e}}{ }^{2}}
$$

$\mathrm{R}_{\mathrm{e}}$ : earth radius

el : is the elevation angle to the spacecraft that defines the EOC.

$\gamma$ : earth central angle, calculated as follows:

$$
\gamma=\sin ^{-1}\left(\frac{\left(\mathrm{R}_{\mathrm{e}}+\mathrm{h}\right) \sin (\theta)}{\mathrm{R}_{\mathrm{e}}}\right)-\theta
$$

Also, the maximum nadir angle $\theta_{\max }$ can be calculated as follows:

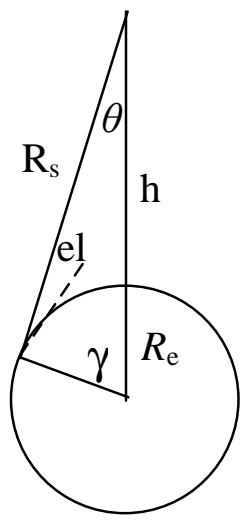

$$
\theta_{\text {max }}=\sin ^{-1}\left(\frac{\cos (\mathrm{el}) \mathrm{R}_{\mathrm{e}}}{\mathrm{R}_{\mathrm{e}}+\mathrm{h}}\right)
$$

And, the general formula of the directivity can be calculated from the antenna field pattern [9], as follows:

$$
\mathrm{D}(\theta, \phi)=\frac{|\mathrm{F}(\theta, \phi)|^{2}}{\frac{1}{4 \pi} \int_{\text {all space }}|\mathrm{F}(\theta, \phi)|^{2} \mathrm{~d} \Omega}
$$

where, $\mathrm{d} \Omega=\sin \theta \mathrm{d} \theta \mathrm{d} \phi$.

The objective is to achieve the optimum Directivity to be close as possible to the desired directivity. Genetic algorithm is used to get the optimum thinned elements to get the optimum directivity at the desired angles without any steering to the beam, that is mean no phase shifters will be needed.

\section{Genetic algorithm optimization:}

Genetic algorithm [10] optimizers are robust stochastic search methods modeled on the principles and concepts of natural selection and evolution. As an optimizer, the powerful heuristic of the GA is effective at solving complex combinatorial and related problems. GA optimizers are particularly effective when the goal is to find an approximate global maximum in a high-dimension, multi-modal function domain.

A genetic algorithm using population decimation, crossover, and mutation was used to 
generate new individuals as shown in Figure (2).

The fitness function is used to determine which of the selected parents better fit to produce offspring for the next generation, to determine which individuals are replaced each generation, and finally get the best chromosome which satisfy the objective.

The fitness or the objective function in this problem is:

$$
\text { fit }=\left|\mathrm{D}(\theta, \phi)-\mathrm{DD}_{\mathrm{r}}(\theta, \phi)\right|
$$

The optimum value of fit equals zero, so the objective is the minimization of fit.

Genes are the excitation coefficients of each element. Each gene represented by one bit, zero or one. Excitation coefficients are real not imaginary so, there is no phase shifters added to the feeding network, which simplify the design. The direction of the maximum is always in the antenna broadside.

Thinning an array to produce the desired directivity is much simpler than the more general problem of nonuniform excitations of the elements [11].

Each chromosome is represented by a 2-D array, and the population is a 3-D array. Crossover as shown in Figure (3), and the probability of mutation is taken 0.1 of the whole population size. Initial population generation, crossover, and mutation techniques have a big effect of the convergence to the optimum solution.

\section{4. simulation results:}

Consider a case of a sun synchronous LEO satellite of altitude $850 \mathrm{Km}$, in a ground track with $\phi$ equals zero. $\theta$ changes from nadir ( $\theta=$ zero) till the EOC [12] which equals $58.4^{\circ}$ for $15^{\circ}$ elevation angle. The directivity is calculated each one degree from nadir till the EOC for $4 \times 4,8 \times 4,8 \times 8$ planar array with half wave rectangular patch element pattern, and $4 \times 4$ planar array with $\cos ^{1.5} \theta$ element pattern.

The excitation coefficients are adjusted to compensate the increasing in free space path losses as the satellite go away from the ground station. The appropriate excitations are achieved by running the built genetic algorithm program to achieve directivity as close as possible to the desired directivity.

Figure (4) shows the desired and achieved directivity through the satellite orbit from nadir till the EOC, the $\cos ^{1.5}(\theta)$ element pattern with $4 \times 4$ planar array configuration gives high directivity near the nadir than the desired by approximately $3 \mathrm{~dB}$ and it is decreased monotonically till $\theta=29^{\circ}$, after that the achieved directivity becomes equal to the desired directivity till $\theta=48^{\circ}$, and after that it decreases till it becomes less than the desired directivity by around $6.4 \mathrm{~dB}$ at the EOC.

Using of rectangular patch element pattern gives good results comparing with $\cos ^{1.5}(\theta)$ one, in case of $4 \times 4$ array with patch element pattern gives approximately the desired 
directivity till $\theta=50^{\circ}$.

The increasing of elements improve the results, so, in case of $8 \times 4$ array it satisfy the desired directivity till $\theta=55^{\circ}$ and decreased by around one $\mathrm{dB}$ at the $\mathrm{EOC}$, finally, in case of 8x8 array it approximately satisfy the desired directivity from nadir till the EOC. Figures (5) through (9) show the normalized far field pattern in case of thinned $4 \times 4$ planar array with rectangular patch elements at different $\theta$, which simulate the movement of the satellite.

Figures (10) through (14) show the normalized far field pattern in case of thinned $4 \times 4$ planar array with $\cos ^{1.5}(\theta)$ elements at different $\theta$.

Figures (15) through (18) show the normalized far field pattern in case of thinned $8 \times 4$ and $8 \times 8$ planar array with rectangular patch elements at different $\theta$,

Tables (1) through (11) show the ON and OFF elements in the array. In the tables, the first top left element represents the element at the origin, the elements in the same row in the y-direction, and the elements in the same column in the x-direction of the planar array.

In many cases, the excitation coefficients which used for certain angle are adequate to be used for other successive angles, for example, the used excitations at $\theta=0$ in case of $8 \times 4$ array is sufficient to get the desired directivity from $\theta=0^{\circ}$ till $\theta=12^{\circ}$, also, the excitation coefficients of $\theta=30^{\circ}$ are adequate till $\theta=36^{\circ}$, and so on, which will simplify the program which will control the feeding network through the movement of the satellite.

Figures (19) and (20) show the convergence to the optimum solution in two different runs to two different cases by using the built genetic algorithm optimizer.

\section{Conclusions:}

Thinning of an $8 \times 4$ rectangular antenna array with half wave rectangular patch element pattern gives good results to achieve the desired directivity to achieve constant link margin during a certain ground track from the nadir till EOC, also an $8 \times 8$ one gives optimum results through all the path.

This simple technique gives good results without need to phase shifters, that's simplify the feeding network. The excitation coefficients which used for certain angle are adequate to be used for other successive angles, that will also simplify the feeding.

GA is a powerful algorithm to achieve a certain objective, it is effective at solving complex combinatorial and related problems. 


\section{References:}

[1] Sherman, K. N., "phased array shaped multi-beam optimization for LEO satellite communications using a genetic algorithm," International Conference on Phased Array Systems and Technology, Dana Point CA, USA, 21-25, pp. 501-504, May (2000)

[2] Randy L. Haupt, “ Thinned Arrays Using Genetic Algorithms," IEEE Trans. Antennas propagation, vol. AP.42, No. 7, pp. 993-999, (1994).

[3] Gary D. Gordon and Walter L. Morgan, principles of Communications Satellites, John Willy \& Sons, Inc. (1993).

[4] J. Michael Johnson and Yahya Rahmat-Samii, "Genetic Algorithms in Engineering Electromagnetics,” IEEE Trans. Antennas propagation, vol. 39, No. 4, pp. 7 21, August (1997).

[5] Randy L. Haupt, "An Introduction to Genetic Algorithms for Electromagnetics," IEEE Trans. Antennas propagation, vol. 37, No. 2, pp. 7 -15, April (1995).

[6] Olen, C. A. and RT. Compton, Jr, “ A numerical pattern synthesis algorithm for arrays,” IEEE Trans. Antennas propagation, vol. 38, No. 10, pp. 1666-1676, (1990).

[7] Zhou, P. Y. P. and M. A. Ingram, "pattern synthesis for arbitrary arrays using an adaptive method,” IEEE Trans. Antennas propagation, vol. 47, No. 5, pp. 862869, (1999).

[8] Robert J. Mailloux, Phased Array Antenna Handbook (2 ${ }^{\text {nd }}$ ed.), Artech House, (2005).

[9] Richard C. Jonson, Antenna Engineering Handbook ( $3^{\text {rd }}$ ed.), McGraw-Hill, (1993).

[10] Hazem H. El-Banna, "Sidelobe Cancellation in antenna arrays for Radar applications,” M.sc. thesis, ch.4.,(2001)

[11] J. Jin, H.L. Wang, “Array patterns synthesizing using genetic algorithm," progress in electromagnetics research symposium, Cambridge, USA, March 26-29, 2006, pp. 64- 68.

[12] E. Jacobs, J. M. Stacey, “Earth Footprints of Satellite Antennas,” IEEE Trans. on Aerospace and Electronic Systems, vol. AES. 7, No. 2, pp. 235-242, March (1971). 


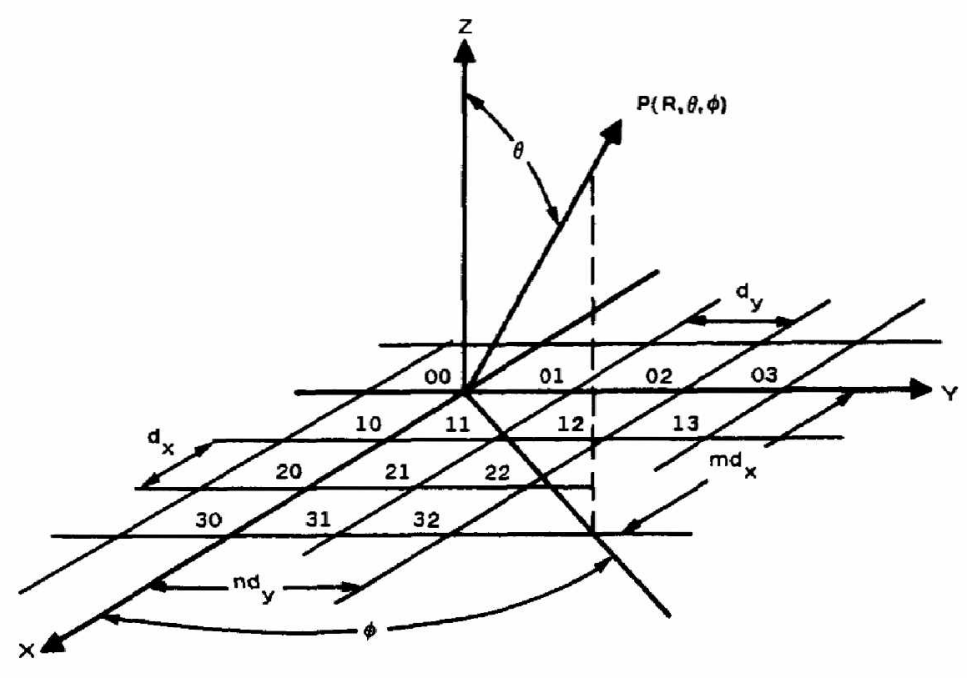

Figure (1): Element geometry of a 2-D planar array.

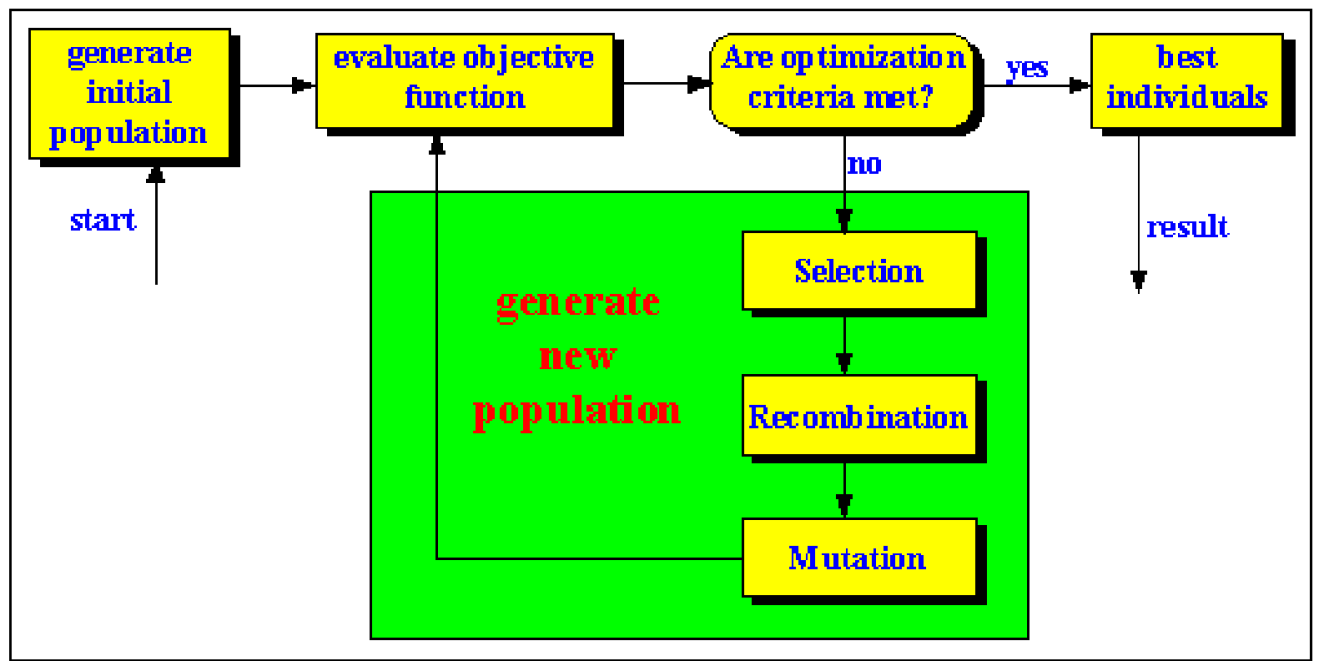

Figure (2): A block diagram of a simple Genetic Algorithm optimizer

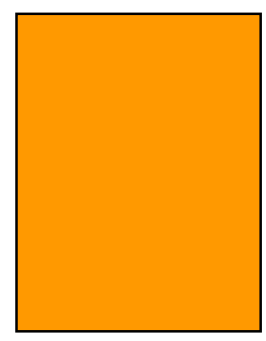

Chromosome \#1

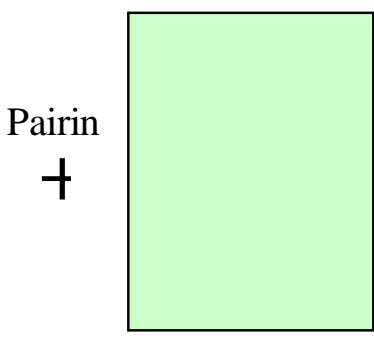

Chromosome \#2

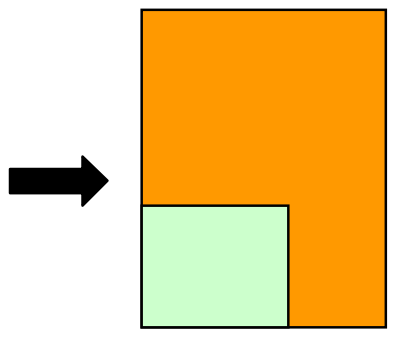

Child \#1

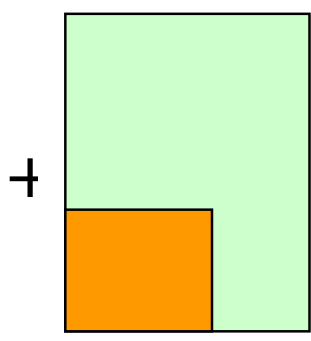

Child \#2

Figure (3): The used technique of crossover in case of planar array chromosomes 


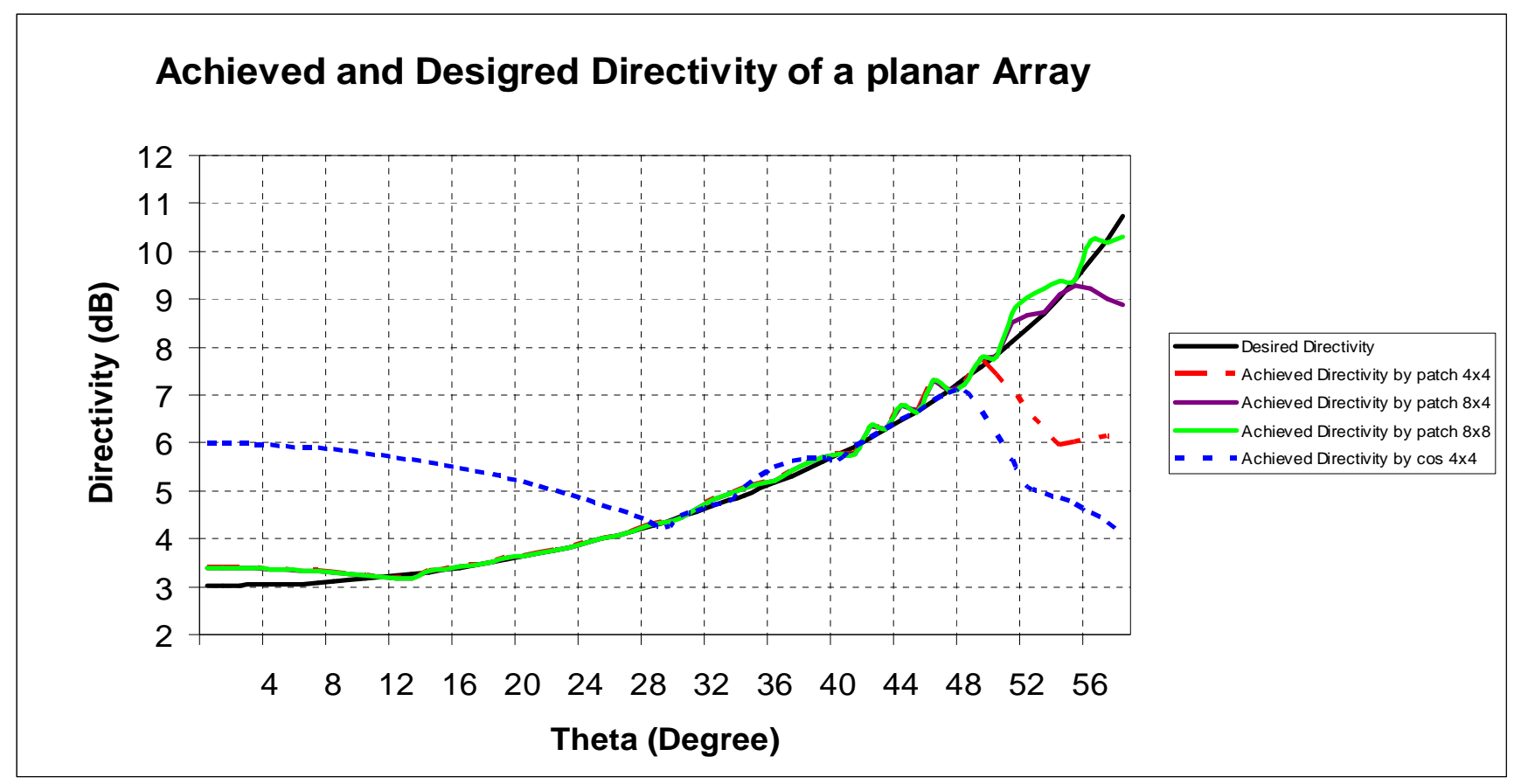

Figure (4): The Achieved and desired Directivity of the planar array

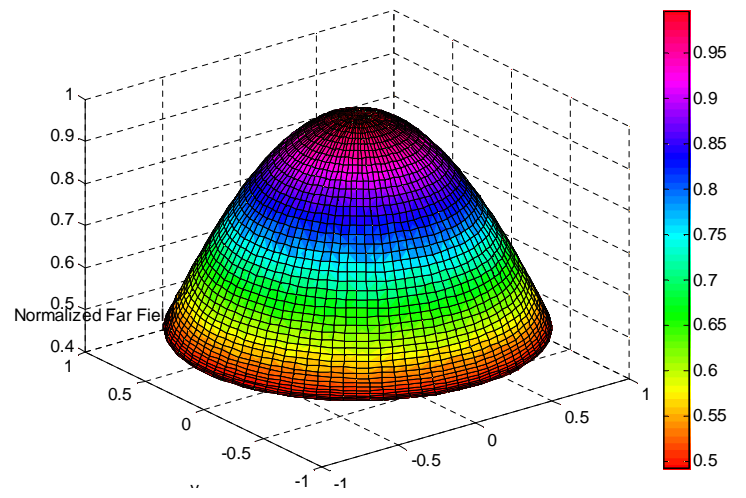

Figure (5): The Normalized far field pattern at theta 0 deg. for $4 x 4$ patch elements array

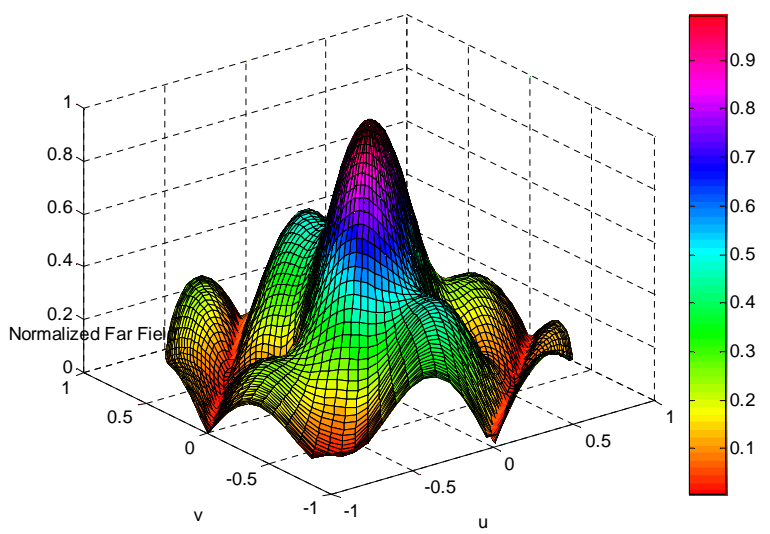

Table (1):The thinned $4 \times 4$ patch elements at theta 0 deg.

\begin{tabular}{|l|l|l|l|}
\hline 0 & 0 & 0 & 0 \\
\hline 0 & 0 & 0 & 1 \\
\hline 0 & 0 & 0 & 0 \\
\hline 0 & 0 & 0 & 0 \\
\hline
\end{tabular}

Table (2):The thinned $4 \times 4$ patch elements at theta $20 \mathrm{deg}$.

\begin{tabular}{|l|l|l|l|}
\hline 0 & 1 & 0 & 1 \\
\hline 1 & 1 & 0 & 0 \\
\hline 1 & 0 & 0 & 0 \\
\hline 1 & 0 & 0 & 0 \\
\hline
\end{tabular}

Figure (6): The Normalized far field pattern at theta 20 deg. for $4 x 4$ patch elements array 


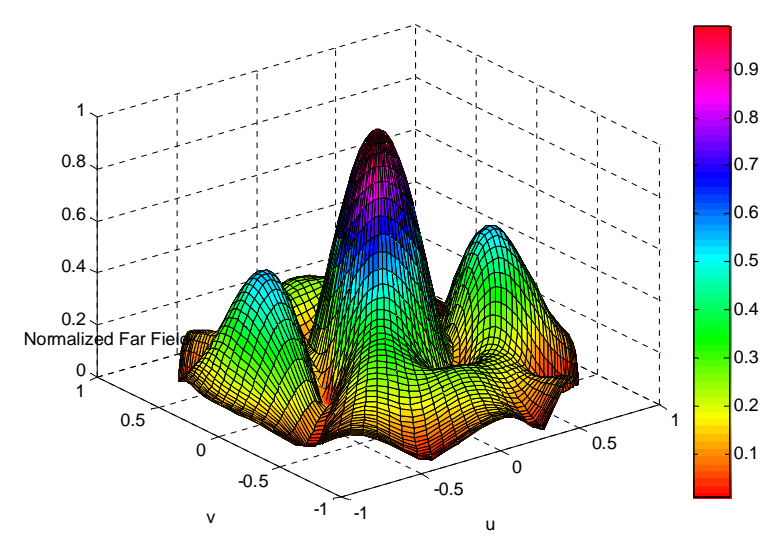

Figure (7): The Normalized far field pattern at theta 40 deg. for $4 x 4$ patch elements array

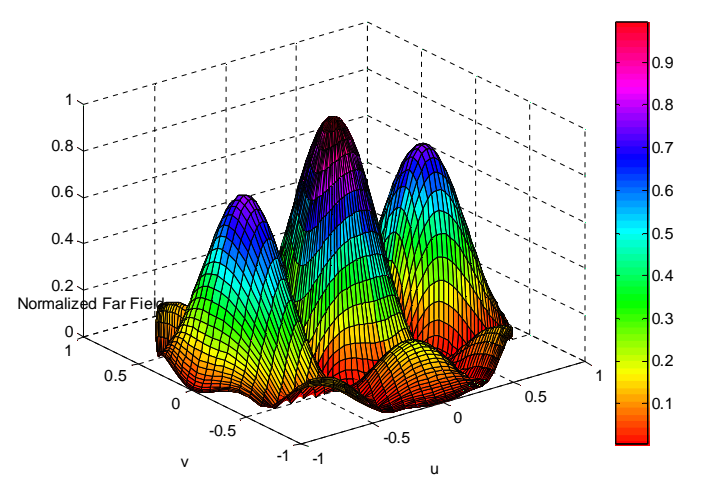

Figure (8): The Normalized far field pattern at theta $50 \mathrm{deg}$. for $4 \times 4$ patch elements array

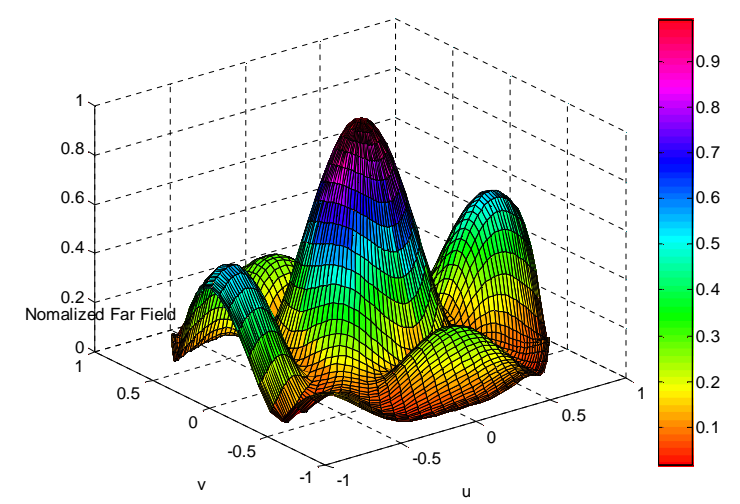

Table (5): The thinned $4 \times 4$ patch elements at theta $58 \mathrm{deg}$.

\begin{tabular}{|l|l|l|l|}
\hline 1 & 1 & 1 & 1 \\
\hline 0 & 0 & 0 & 0 \\
\hline 1 & 1 & 1 & 1 \\
\hline 0 & 0 & 0 & 0 \\
\hline
\end{tabular}

Figure (9): The Normalized far field pattern at theta 58 deg. for $4 x 4$ patch elements array 

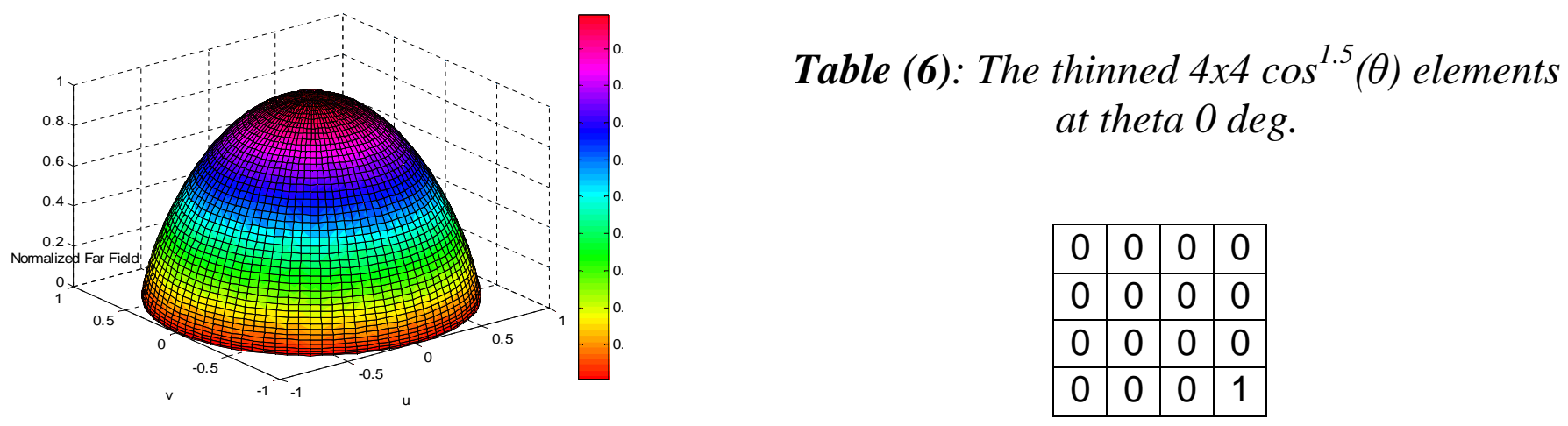

Figure (10): The Normalized far field pattern at theta 0 deg. for $4 x 4 \cos ^{1.5}(\theta)$ elements array

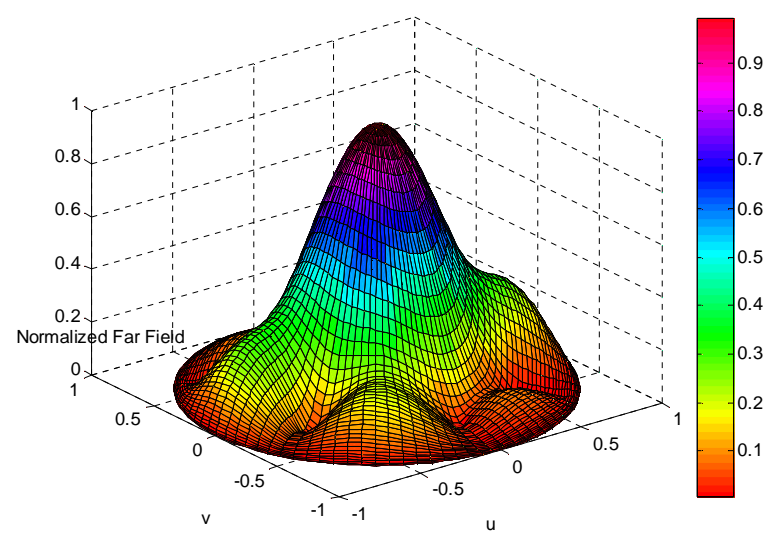

Table (7): The thinned $4 \times 4 \cos ^{1.5}(\theta)$ elements at theta $30 \mathrm{deg}$.

\begin{tabular}{|l|l|l|l|}
\hline 1 & 1 & 1 & 1 \\
\hline 0 & 0 & 1 & 0 \\
\hline 0 & 1 & 0 & 0 \\
\hline 0 & 0 & 0 & 0 \\
\hline
\end{tabular}

Figure (11): The Normalized far field pattern at theta $30 \mathrm{deg}$. for $4 x 4 \cos ^{1.5}(\theta)$ elements array

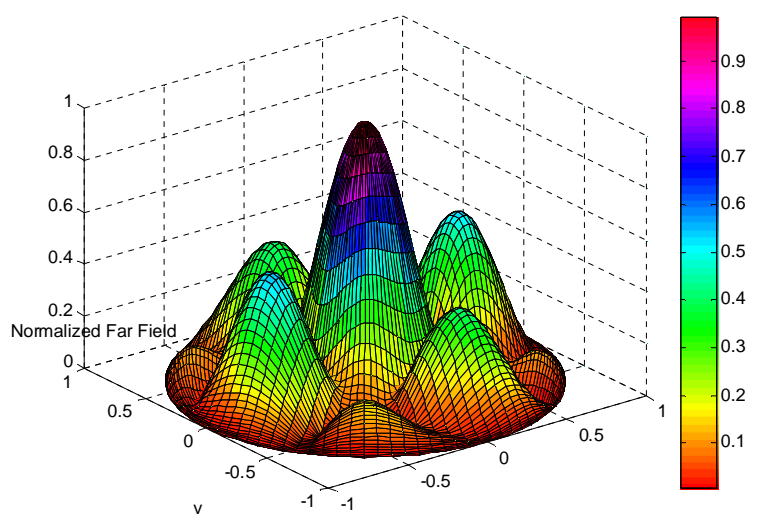

Table (8): The thinned $4 x 4 \cos ^{1.5}(\theta)$ elements at theta 45 deg.

\begin{tabular}{|l|l|l|l|}
\hline 1 & 0 & 1 & 1 \\
\hline 0 & 0 & 0 & 0 \\
\hline 0 & 0 & 0 & 1 \\
\hline 1 & 1 & 0 & 1 \\
\hline
\end{tabular}

Figure (12): The Normalized far field pattern at theta 45 deg. for $4 x 4 \cos ^{1.5}(\theta)$ elements array 


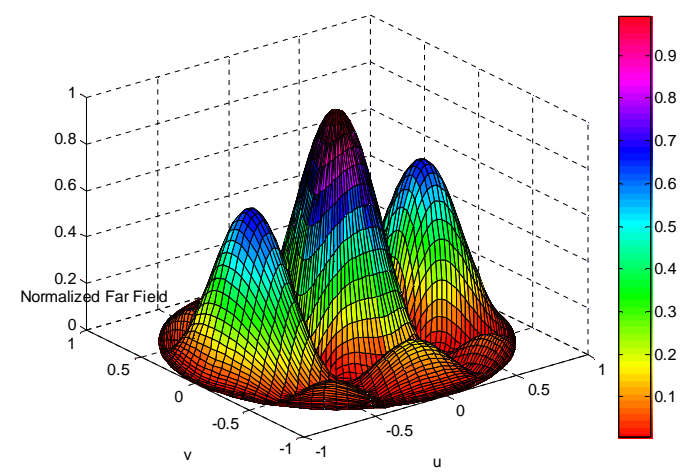

Table (8): The thinned $4 x 4 \cos ^{1.5}(\theta)$ elements at theta $50 \mathrm{deg}$.

\begin{tabular}{|l|l|l|l|}
\hline 1 & 1 & 1 & 1 \\
\hline 0 & 0 & 0 & 0 \\
\hline 0 & 0 & 0 & 0 \\
\hline 1 & 1 & 1 & 1 \\
\hline
\end{tabular}

Figure (13): The Normalized far field pattern at theta 50 deg. for $4 x 4 \cos ^{1.5}(\theta)$ elements array

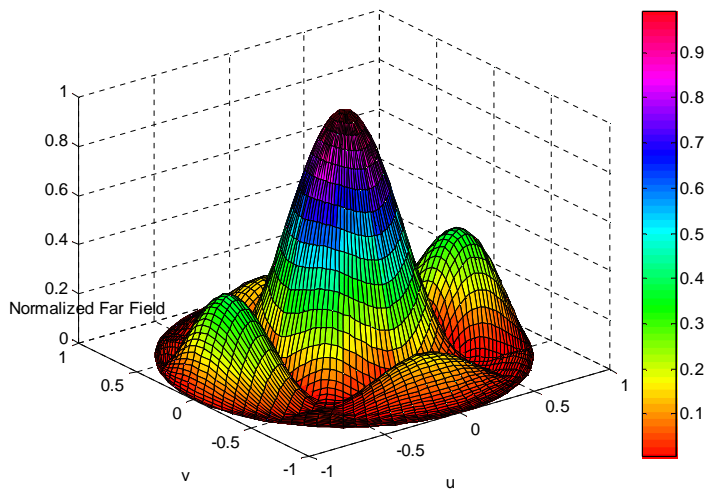

Figure (14): The Normalized far field pattern at theta 58 deg. for $4 x 4 \cos ^{1.5}(\theta)$ elements array

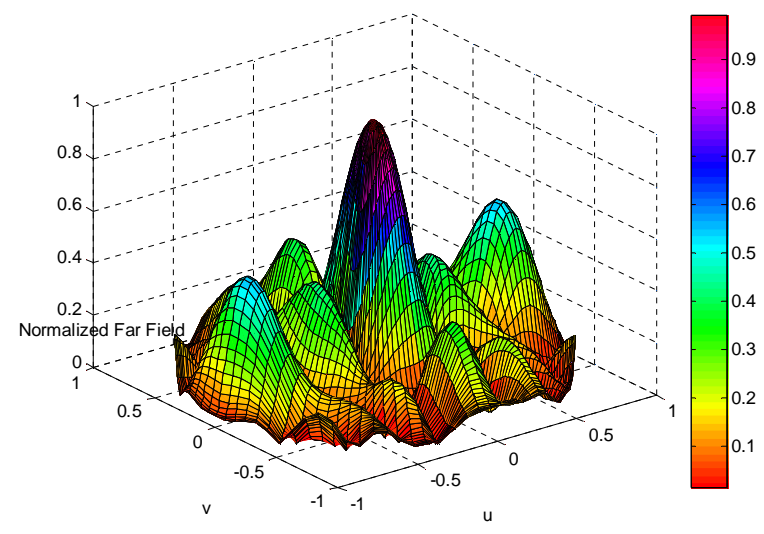

Figure (15): The Normalized far field pattern at theta 50 deg. for $8 \times 4$ patch elements array
Table (8): The thinned $4 x 4 \cos ^{1.5}(\theta)$ elements at theta $58 \mathrm{deg}$.

\begin{tabular}{|l|l|l|l|}
\hline 0 & 0 & 0 & 0 \\
\hline 1 & 1 & 1 & 1 \\
\hline 0 & 0 & 0 & 0 \\
\hline 1 & 1 & 1 & 1 \\
\hline
\end{tabular}

Table (8): The thinned $8 \times 4$ patch elements at theta $50 \mathrm{deg}$.

\begin{tabular}{|l|l|l|l|}
\hline 1 & 0 & 0 & 1 \\
\hline 0 & 0 & 0 & 0 \\
\hline 1 & 1 & 1 & 1 \\
\hline 0 & 0 & 0 & 0 \\
\hline 0 & 0 & 0 & 0 \\
\hline 1 & 0 & 1 & 0 \\
\hline 0 & 0 & 0 & 0 \\
\hline 1 & 0 & 1 & 1 \\
\hline
\end{tabular}




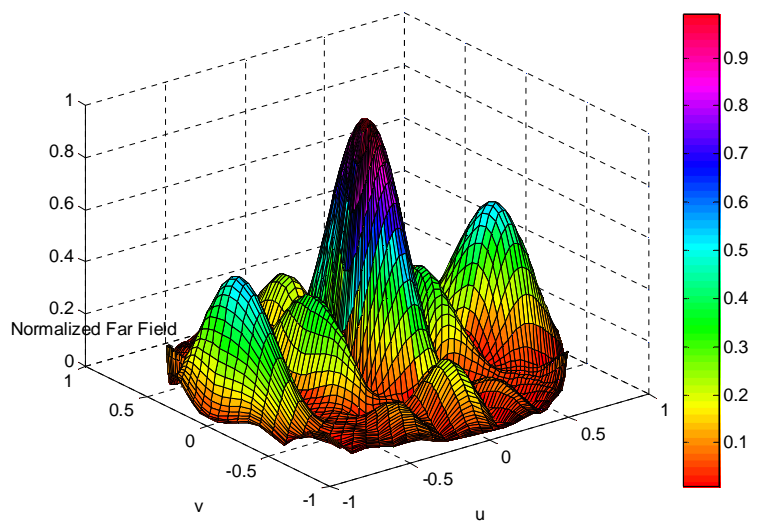

Figure (16): The Normalized far field pattern at theta 58 deg for $8 \times 4$ patch elements array

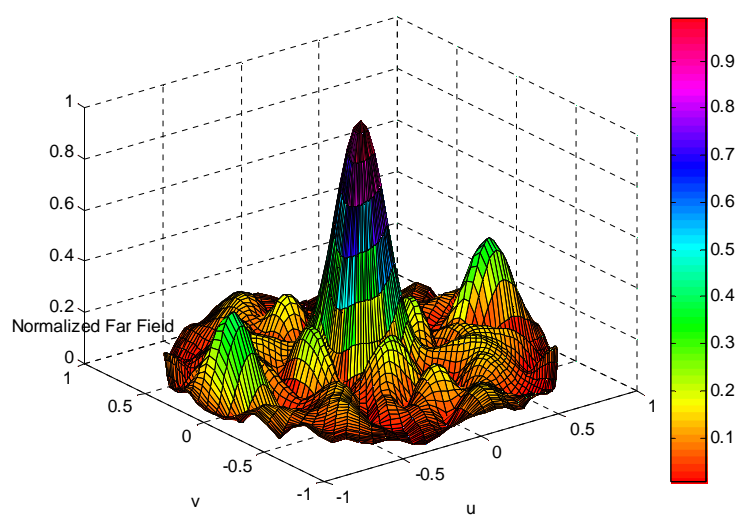

Figure (17): The Normalized far field pattern at theta 55 deg for $8 \times 8$ patch elements array

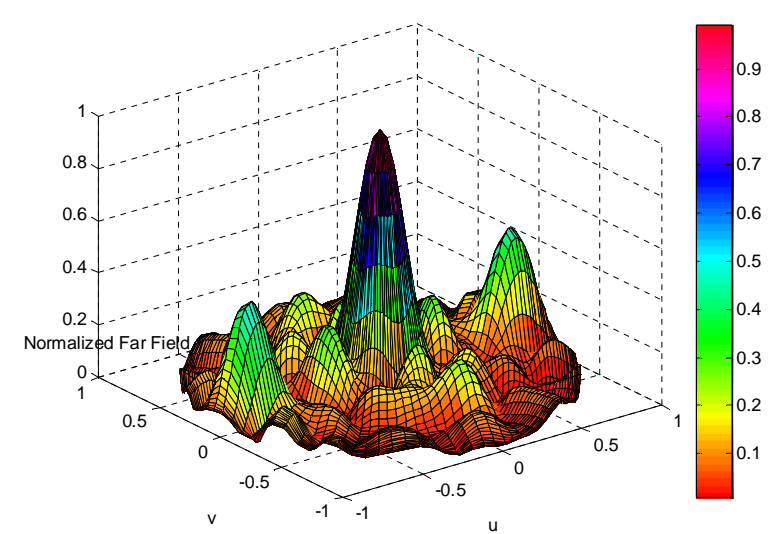

Figure (18): The Normalized far field pattern at theta 58 deg for $8 x 8$ patch elements array
Table (9): The thinned $8 \times 4$ patch elements at theta 58 deg.

\begin{tabular}{|l|l|l|l|}
\hline 1 & 1 & 1 & 1 \\
\hline 0 & 0 & 0 & 0 \\
\hline 1 & 1 & 1 & 1 \\
\hline 0 & 0 & 0 & 0 \\
\hline 0 & 0 & 0 & 0 \\
\hline 1 & 1 & 1 & 1 \\
\hline 0 & 0 & 0 & 0 \\
\hline 1 & 1 & 1 & 1 \\
\hline
\end{tabular}

Table (10): The thinned $8 x 8$ patch elements at theta $55 \mathrm{deg}$.

\begin{tabular}{|l|l|l|l|l|l|l|l|}
\hline 1 & 1 & 1 & 1 & 1 & 0 & 1 & 1 \\
\hline 0 & 0 & 0 & 0 & 0 & 0 & 1 & 0 \\
\hline 1 & 1 & 1 & 1 & 1 & 0 & 1 & 1 \\
\hline 0 & 0 & 0 & 0 & 1 & 1 & 0 & 0 \\
\hline 0 & 0 & 0 & 0 & 1 & 0 & 1 & 0 \\
\hline 1 & 0 & 1 & 0 & 0 & 1 & 1 & 1 \\
\hline 0 & 0 & 0 & 0 & 0 & 0 & 0 & 0 \\
\hline 1 & 0 & 0 & 1 & 1 & 1 & 0 & 1 \\
\hline
\end{tabular}

Table (11): The thinned $8 x 8$ patch elements at theta 58 deg.

\begin{tabular}{|l|l|l|l|l|l|l|l|}
\hline 1 & 1 & 1 & 1 & 0 & 1 & 1 & 1 \\
\hline 0 & 0 & 0 & 0 & 0 & 0 & 0 & 0 \\
\hline 1 & 1 & 1 & 1 & 1 & 1 & 1 & 1 \\
\hline 0 & 0 & 0 & 0 & 1 & 0 & 0 & 0 \\
\hline 1 & 0 & 0 & 0 & 0 & 0 & 0 & 0 \\
\hline 1 & 0 & 0 & 1 & 1 & 1 & 0 & 1 \\
\hline 0 & 0 & 0 & 0 & 0 & 0 & 0 & 0 \\
\hline 1 & 1 & 0 & 0 & 1 & 0 & 1 & 1 \\
\hline
\end{tabular}




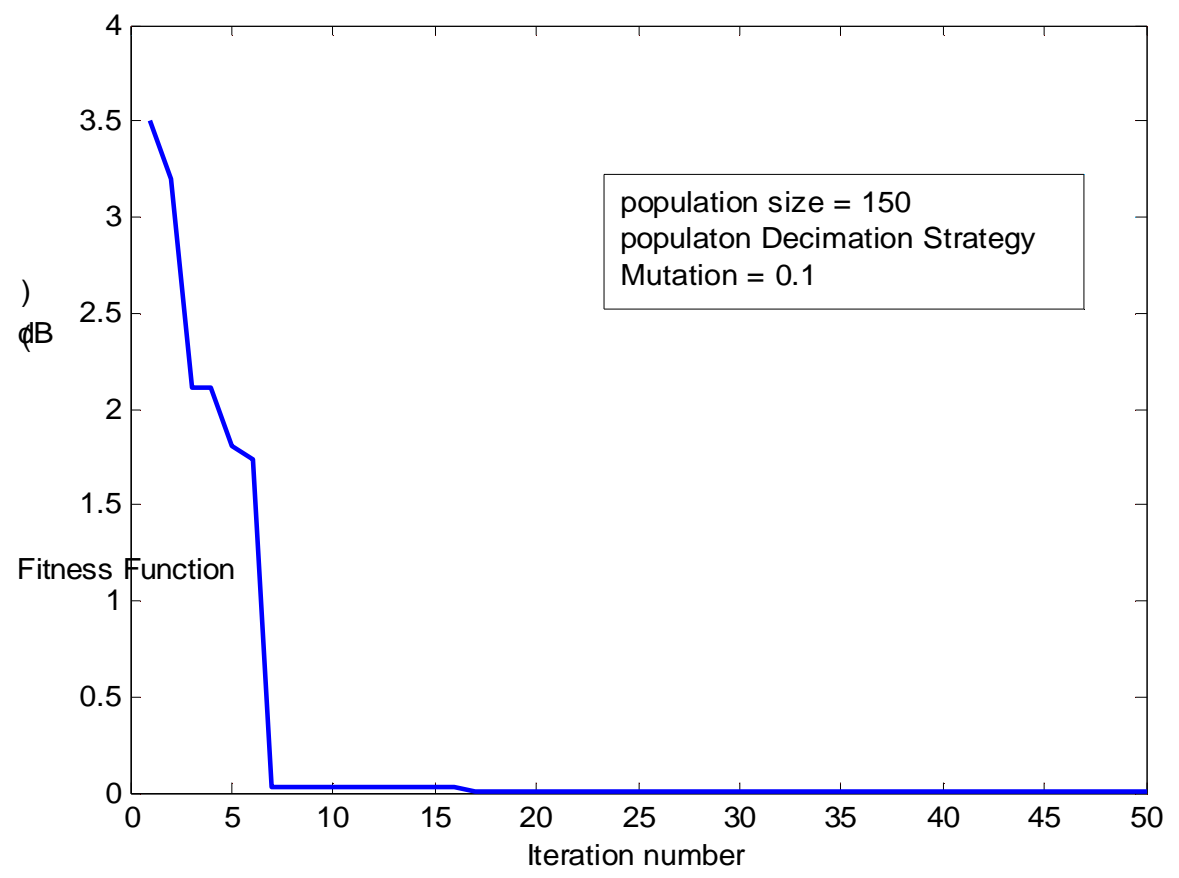

Figure (19): The Genetic algorithm convergence at theta 55 for $8 x 8$ patch elements array

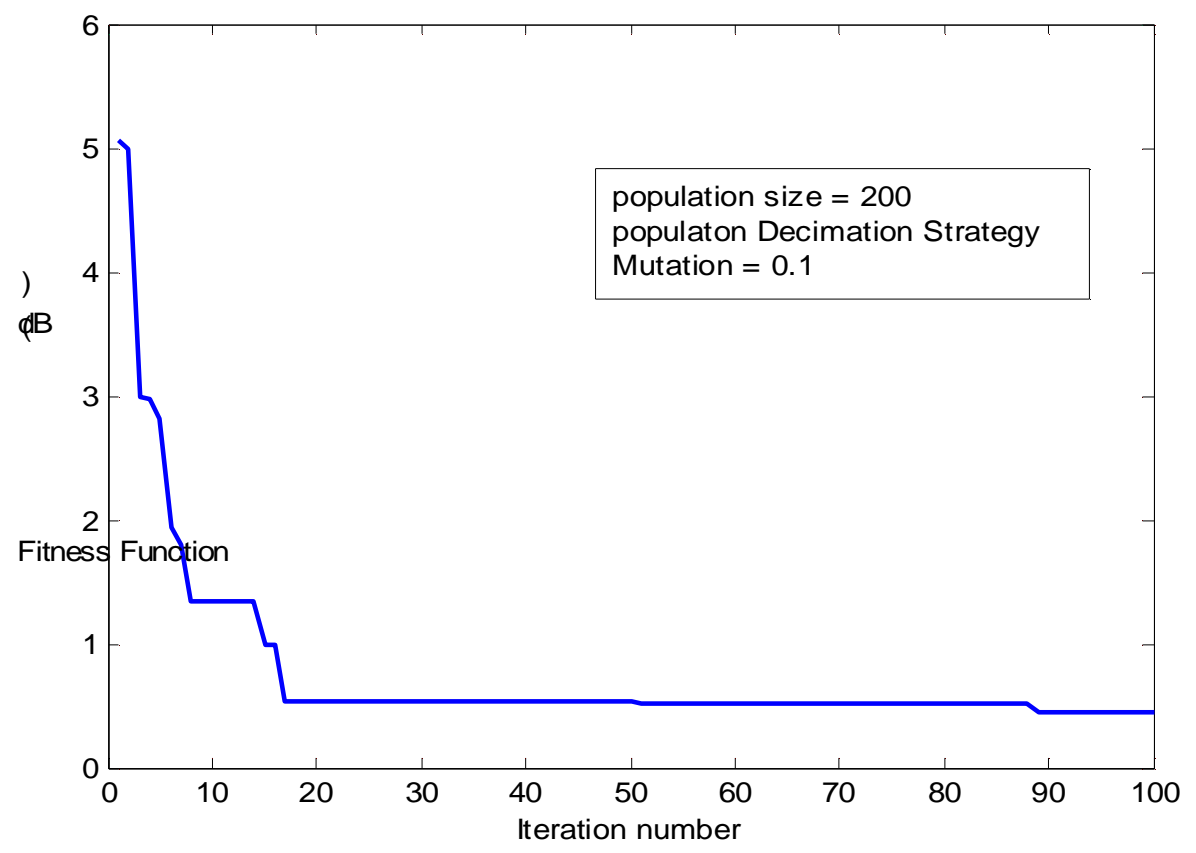

Figure (20): The Genetic algorithm convergence at theta 58 deg for $8 x 8$ patch elements array 


\begin{tabular}{|l|l|l|l|l|l|l|l|}
\hline 00 & 01 & 02 & 03 & 04 & 05 & 06 & 07 \\
\hline 10 & 11 & 12 & 13 & 14 & 15 & 16 & 17 \\
\hline 20 & 21 & 22 & 23 & 24 & 25 & 26 & 27 \\
\hline 30 & 31 & 32 & 33 & 34 & 35 & 36 & 37 \\
\hline 40 & 41 & 42 & 43 & 44 & 45 & 46 & 47 \\
\hline 50 & 51 & 52 & 53 & 54 & 55 & 56 & 57 \\
\hline 60 & 61 & 62 & 63 & 64 & 65 & 66 & 67 \\
\hline 70 & 71 & 72 & 73 & 74 & 75 & 76 & 77 \\
\hline
\end{tabular}

